\title{
Formation of complex two-dimensional dissipative solitons via spontaneous symmetry breaking
}

\author{
V. Skarka, ${ }^{1,2,3,{ }^{*}}$ N. B. Aleksić, ${ }^{2,3}$ M. Lekić, ${ }^{2}$ B. N. Aleksić, ${ }^{2,3}$ B. A. Malomed, ${ }^{4}$ D. Mihalache, ${ }^{5}$ and H. Leblond ${ }^{1}$ \\ ${ }^{1}$ Laboratoire de Photonique d'Angers, EA 4464, Université d'Angers, 2 Boulevard Lavoisier, 49045 Angers Cedex 01, France \\ ${ }^{2}$ Institute of Physics, University of Belgrade, 11000 Belgrade, Serbia \\ ${ }^{3}$ Texas A\&M University at Qatar, PO Box 23874, Doha, Qatar \\ ${ }^{4}$ Department of Physical Electronics, Faculty of Engineering, Tel Aviv University, Tel Aviv 69978, Israel \\ ${ }^{5}$ Horia Hulubei National Institute for Physics and Nuclear Engineering, 407 Atomistilor, Magurele-Bucharest, 077125, Romania
}

(Received 12 May 2013; published 25 August 2014)

\begin{abstract}
We propose a complex Ginzburg-Landau equation (CGLE) with localized linear gain as a two-dimensional model for pattern formation proceeding via spontaneous breaking of the axial symmetry. Starting from steady-state solutions produced by an extended variational approximation, simulations of the CGLE generate a vast class of robust solitary structures. These are varieties of asymmetric rotating vortices carrying the topological charge (TC), and four- to ten-pointed revolving stars, whose angular momentum is decoupled from the TC. The fourand five-pointed stars feature a cyclic change of their structure in the course of the rotation.
\end{abstract}

DOI: 10.1103/PhysRevA.90.023845

PACS number(s): 42.65.Tg, 42.65.Sf, 47.20.Ky

\section{INTRODUCTION}

The generation of self-organized dissipative structures in nonlinear systems is driven by external energy and/or matter supplies [1]. Various species of self-trapped localized structures are represented by dissipative solitons acting as attractors [2,3]. The self-organization is based on the balance of antagonistic effects, with gain compensating losses, and nonlinearity-induced self-contraction arresting the linear diffraction and/or dispersion. The ensuing formation of vast varieties of patterns is apparently spontaneous, and in many cases its origins are not yet understood well, in spite of the great deal of work done on this subject. Generically, the pattern formation proceeds via spontaneous breaking of an underlying continuous symmetry, followed by the emergence of novel forms which feature reduced symmetries [4]. Since the pioneering work of Turing [5], many works have been dealing with models for spontaneous pattern formation in diverse settings; see, e.g., Refs. [6,7].

Complex Ginzburg-Landau equations (CGLEs) constitute a class of ubiquitous models to describe the generation of dissipative-solitonic structures in plenty of systems ranging from nanophotonics, plasmonics, nonlinear optics, fluids, and plasmas through superconductivity, superfluidity, quantum field theory, and biological systems [2,3,8-11]. The great deal of work done in this field has demonstrated cogently that CGLEs are appropriate models for studying the spontaneous pattern formation per se.

In this work, we demonstrate that the above-mentioned crucially important aspect of the pattern formation, viz., the spontaneous breaking of the continuous symmetry, leading to the emergence of localized structures featuring reduced symmetries, may be adequately modeled by a suitably chosen two-dimensional (2D) CGLE with the competing cubicquintic (CQ) nonlinear terms. We resort to the synergy of the variational approximation (VA) and parallelized numerical simulations to demonstrate spontaneous formation of previously unexplored solitonic structures, which have diverse

\footnotetext{
*vladimir.skarka@univ-angers.fr
}

counterparts in nature. Using the CGLE with a spatially modulated linear loss, which features a minimum at the center, we have previously demonstrated that vortices may spontaneously evolve into stably rotating ellipsoidal or crescent vortical structures [12]. The CGLE model developed in the present work offers a vast potential for modeling transitions between different types of spontaneously established patterns, through the generation a broad class of localized states, such as periodically metamorphosing and rotating four- to ten-pointed stars, which resemble complex natural objects, but were not produced by previously studied models.

As mentioned above, the dissipative-soliton pattern formation is the result of the concurrent balance between losses and gain, and between diffraction and cubic self-focusing, which must be supplemented by the quintic self-defocusing, to prevent the collapse in the $2 \mathrm{D}$ geometry. The creation of $(2+1) \mathrm{D}$ optical solitons (two transverse coordinates $x$ and $y$, with +1 standing for the propagation distance $z$, which plays the role of the evolutional variable) in a CQ medium has been recently directly demonstrated in an experiment [13]. Dissipative solitons have been found in many varieties of CGLEs [2,3,8,14-16]. In particular, $(2+1) \mathrm{D}$ solitons with embedded vorticity $m$, featuring zero intensity at the center, carry the angular momentum, $M=m P$, where $P$ is their total power (norm); see Eq. (3) below [17]. As a result of the spontaneous pattern change, the intrinsic angular momentum can transmute into explicit rotation of solitonic patterns, as shown in the movie in the Supplemental Material [18] (examples of this are known, e.g., in the form of azimuthons [19] and vortex gap solitons [20]).

\section{MODEL FOR SELF-ORGANIZED PATTERN FORMATION}

The present model is based on the $(2+1) \mathrm{D}$ CGLE with the CQ nonlinearity that governs the evolution of wave amplitude $E(x, y, z)$ in the nonlinear medium:

$$
\begin{aligned}
& i E_{z}+(1 / 2)\left(E_{x x}+E_{y y}\right)+(1-i \varepsilon)|E|^{2} E-(v-i \mu)|E|^{4} E \\
& \quad=i g(r) E
\end{aligned}
$$


where positive coefficients $\varepsilon, \mu$, and $v$, account, respectively, for the cubic gain, quintic loss, and quintic saturation of the cubic self-focusing. A crucially important ingredient of the model is represented by an "iceberg of the gain," $g(r)=$ $\gamma-\Gamma r^{2}$ (with radial variable $r=\sqrt{x^{2}+y^{2}}$, gain amplitude $\gamma>0$, and gain curvature $\Gamma>0$ ) protruding above the surface of the "loss sea," contrary to the above-mentioned model with the "submerged iceberg," where the main control parameter $\gamma$ is negative [12]. A straightforward physical implementation of Eq. (1) is provided by optically pumped laser cavities (especially end-pump solid-state ones). The pumped beam is focused in order to increase the gain, hence the gain is localized [21]. The transverse localization of the laser beam known as "gain guiding" is used in titanium-sapphire, solid-state, Raman, free-electron, and x-ray lasers [22]. The pump beam is typically Gaussian with intensity $I(r)=I_{0} \exp \left(-r^{2} / R^{2}\right)$, where $R$ is its waist. The gain curvature $\Gamma \propto 1 / R^{2}$ depends mainly on $R$, so that the localized gain is determined by the Taylor expansion of intensity $I(r)$, which makes the model generic [21-23]. The laser cavity can be adjusted by selecting parameters of the saturable absorber [2,8,9,12,21-23]. Thus, the pattern-formation scenarios reported below can be directly realized in the lasers, as well as in other self-organized systems.

Barring rare exceptions [24], the CGLEs, due to their complexity, do not admit exact solutions. Nevertheless, an analytical approximation for dissipative solitons has been developed using the VA adapted to dissipative systems [11,12], see also Ref. [25]. The VA makes use of the following Gaussian trial function representing the electric field of an axisymmetric Gaussian laser beam with vorticity (topological charge, TC) $m=1$ :

$$
E=A(r / R) \exp \left[-r^{2} /\left(2 R^{2}\right)+i C r^{2}+i \theta+i \Psi\right],
$$

where amplitude $A$, radius $R$, wave-front curvature $C$, and phase $\Psi$ have to be optimized. $\theta$ is the angular coordinate. The total power and angular momentum of the vortex are, respectively,

$$
\begin{aligned}
P & =\int_{0}^{\infty} r d r \int_{0}^{2 \pi} d \theta|E(r, \theta)|^{2}=\pi A^{2} R^{2}, \\
M & =i \int_{0}^{\infty} r d r \int_{0}^{2 \pi} d \theta \frac{\partial E^{*}}{\partial \theta} E .
\end{aligned}
$$

Skipping straightforward details, the following system of evolution equations is produced by the VA (although formally similar to the one derived in Ref. [12], it produces essentially different results, as shown below):

$$
\begin{aligned}
& d A / d z=\gamma A-\Gamma R^{2} A+5 \varepsilon A^{3} / 16-8 \mu A^{5} / 81-2 A C, \\
& d R / d z=2 C R-\Gamma R^{3}-\varepsilon A^{2} R / 16+2 \mu A^{4} R / 81, \\
& d C / d z=-2 C^{2}+1 /\left(2 R^{4}\right)-A^{2} /\left(16 R^{2}\right)+2 \nu A^{4} /\left(81 R^{2}\right),
\end{aligned}
$$

$d \Psi / d z=-2 / R^{2}+3 A^{2} / 8-10 v A^{4} / 81$,

with Eq. (7) decoupled from (4)-(6). Fixed points (FPs) of these equations correspond to steady-state solutions with a small wave-front curvature. Setting $d R / d z=0$ in Eq. (5) leads to $C=\Gamma R^{2} / 2+\varepsilon A^{2} / 32-\mu A^{4} / 81$. In Eq. (6) with $d C / d z=0$, small $C^{2}$ may be neglected giving $R^{2}=8\left(A^{2}-32 v A^{4} / 81\right)^{-1}$. The remaining relation for the FP, following from Eqs. (4) with $d A / d z=0$, is $\gamma+\varepsilon A^{2} / 4-2 \mu A^{4} / 27=2 \Gamma R^{2}$, which gives rise to two physically relevant steady-state solutions for amplitude $A$ (solutions for which both $A$ and $R$ are real and positive). According to general principles of the analysis of dissipative systems [24,11], the solution with larger $A$ may be stable, while the one with smaller $A$ is always unstable. The former solution satisfies condition $C<0$, which is necessary for the simultaneous stable balance of the diffraction and CQ nonlinearity, and of the gain and loss, thus rendering the dissipative solitons stable stationary modes [14,26].

The linear stability analysis of the FPs against small perturbations of amplitude, radius, and wave-front curvature within the framework of Eqs. (4)-(6) was performed via the computation of eigenvalues produced by the respective equation $\lambda^{3}+\alpha_{1} \lambda^{2}+\alpha_{2} \lambda-\alpha_{3}=0$,

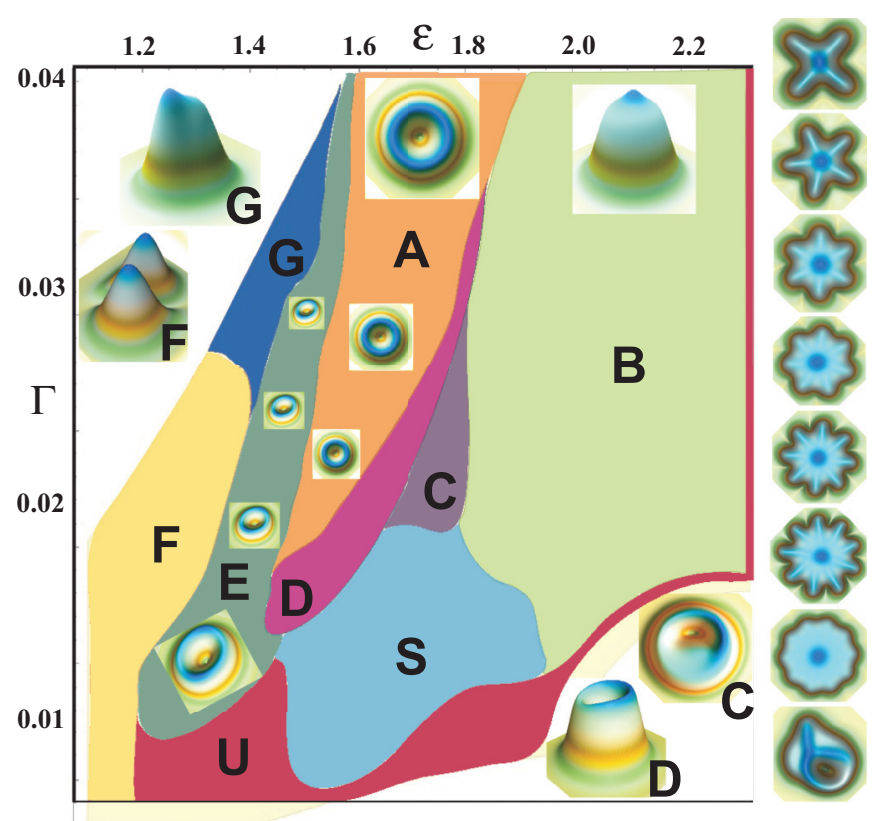

FIG. 1. (Color online) Stability domains produced by the VAgenerated fixed points, which are used as the input for direct simulations of Eq. (1), in the plane of the nonlinear-gain strength, $\varepsilon$, and the linear-gain curvature, $\Gamma$ (dimensionless units). Stable axisymmetric vortices are established in region $\mathrm{A}$. In region $\mathrm{B}$, the spontaneous symmetry breaking expels the vortical phase dislocation, resulting in the transition to a bell-shaped axisymmetric mode without the central crater. In intermediate area $\mathrm{C}$, the vortex mutates into a rotating crescent-shaped soliton that fills only half of the original crater, thus breaking the inner circular symmetry. In region $\mathrm{D}$, the vortex crater gets slanted but remains centrosymmetric. In area E, the modulational instability spontaneously breaks the axial symmetry, converting the circular vortex into a rotating elliptic one. In area $\mathrm{F}$, the ring splits into two bell-shaped fragments. For larger $\Gamma$ in region $\mathrm{G}$, they merge into a stable double-hump shape. In the region of star-shaped patterns, $\mathrm{S}$, small variations of $\Gamma$ and $\varepsilon$ result in the self-trapping of revolving four-, five-, six-, seven-, eight-, nine-, and ten-pointed stars with zero TC (examples are shown on the right). The strongly asymmetric steadily rotating "cobra" pattern, shown at the bottom of the right column, appears in interstices between different regions. Structure fails to form only in region $\mathrm{U}$. 
stable FPs being identified by the Routh-Hurwitz conditions [11]: $\alpha_{1} \equiv 2 \Gamma R^{2}-5 \varepsilon A^{2} / 8+32 \mu A^{4} / 81>0$, $\alpha_{2} \equiv 8 v A^{4} R^{-2} / 81-\Gamma A^{2} R^{2}\left(3 \varepsilon / 2-80 \mu A^{2} / 81\right)>0, \alpha_{3} \equiv$ $\left(A^{2}-16 R^{-2}\right) \Gamma-\left(\varepsilon-16 \mu A^{2} / 9\right) A^{2} R^{-4}>0$, and $\alpha_{1} \alpha_{2}-$ $\alpha_{3}>0$. They cover the entire area charted in Fig. 1 in the plane of the nonlinear-gain and linear-gain curvature parameters, $\varepsilon$ and $\Gamma$, which are most essential for the control of the patterns and transitions between them. For effective saturable absorbers in laser cavities, such as those created by means of a Kerr lens, nonlinear polarization rotation, or an appropriate dopant, these parameters can be easily adjusted in the experiment [21-23]. For instance, different concentrations of the rhodamine dye in ethanol, used as a dopant, can be used in order to recover different values of the nonlinear-gain parameter, $\varepsilon$ in Fig. 1. The linear-gain curvature parameters $\Gamma$ can be adjusted to fit values in Fig. 1 by changing the pump-beam intensity. Other coefficients are fixed here as $v=0.4, \mu=1.4$, and $\gamma=0.08$, which adequately represent the generic situation (varying these parameters does not entail essential changes). Steady-state solutions do not exist in the white corners of Fig. 1.

Next, Gaussian electric field (2) with parameters of the stable FP adopted by the VA was used as the input for parallelized [27] simulations of Eq. (1). As a result, a variety of stable patterns have been generated, some of them similar to the one assumed by Eq. (2), and some completely different, as summarized in Fig. 1.

\section{SELF-GENERATED DISSIPATIVE VORTEX SOLITONS}

In area $\mathrm{A}$ of Fig. 1, stable axisymmetric vortex solitons (shown by insets) quickly self-trap by $z=10$. In fact, only in this area does the model give rise to the simple vortex solitons assumed by Eq. (2), while in other domains unique patterns appear. First, in "filamentation" region F, the modulation instability breaks the vortices into two fragments (see the inset also labeled F), as the total power $P$ given by Eq. (3) is not strong enough to keep the vortex structure stable. In contrast, in area $\mathrm{G}$, the power and strength of the pinning to the localized gain (provided by the pump laser) are much larger, leading to coalescence of the fragments into a revolving double-hump soliton above an "effective threshold" for the pattern formation (see movie [18]); cf. Ref. [17]. An example of this stable pattern is shown in inset $\mathrm{G}$.

In region $\mathrm{E}$ adjacent to $\mathrm{F}$, with larger cubic-gain coefficient $\varepsilon$, the modulational instability is not strong enough to destroy the vortex, but it breaks the axial symmetry and deforms it into a stable elliptic rotating vortex. Adjacent to A, but on the opposite side (region D), the vortex remains centrosymmetric, but with a slanted shape (see inset D in Fig. 1). At still larger $\varepsilon$, in region $\mathrm{C}$, the circular symmetry of the vortex is broken by its transformation into a crescent mode, featuring a half-filled vortical ring.

The common feature of these modes is that they start the self-organization as axisymmetric vortices, and the spontaneous symmetry breaking sets in after a period of a quasistable evolution, which may last for up to thousands of propagation units (unless a symmetry-breaking perturbation is added initially). The robustness of the finally established symmetryreduced modes has been confirmed by the propagation over
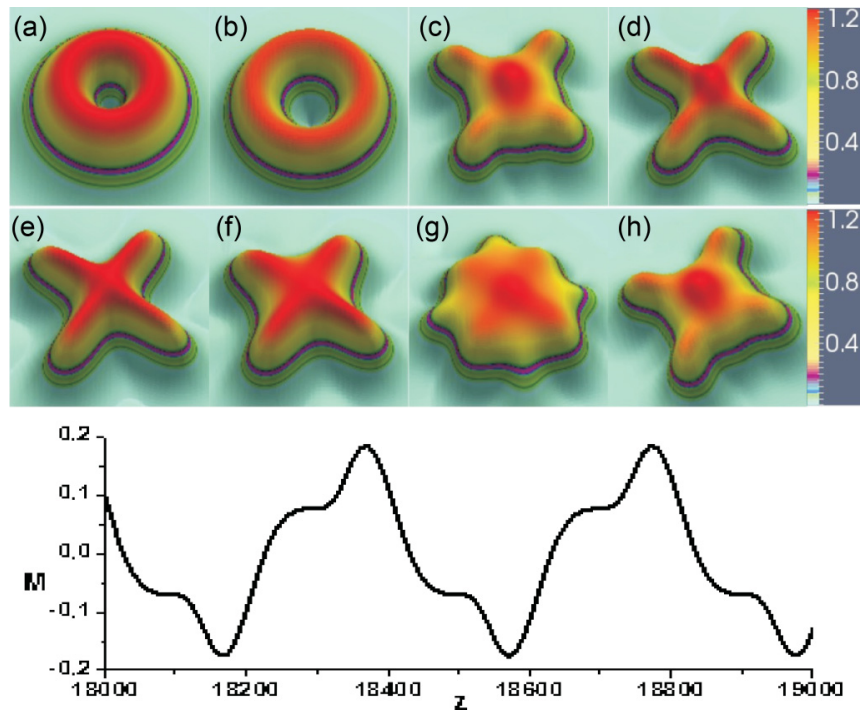

FIG. 2. (Color online) The spontaneous formation of fourpointed patterns. The input ring structure (a) evolves into a vortex soliton at $z \approx 40$ (b), whose spontaneous symmetry breaking in (c) produces a Celtic-cross structure (at $z \approx 1400$ ) that subsequently transmutes into other varieties of cross patterns from $z \approx 1440$ until $z \approx 1560(\mathrm{~d})-(\mathrm{g})$. (h) After a half period $\left(T_{\mathrm{M}} \approx 400\right)$, a rotated Celtic-cross reappears at $z \approx 1600$. Graph $M$ shows oscillations of the angular momentum between $M=-0.18$ and $M=+0.18$ (dimensionless units).

$z>20000$. On the other hand, small gain curvature $\Gamma$ cannot prevent quick destruction of vortices in the bottom region $\mathrm{U}$ ("unstable") of Fig. 1.

At larger values of $\Gamma$ and $\varepsilon$ (in region $B$ ), the original mode undergoes a faster transient evolution, lasting for several hundreds of units, in the form of oscillating breathers, before losing the intrinsic vorticity. Thus, a spontaneous change of the shape occurs, expelling the phase dislocation [see also Fig. 4(a)] and filling the corresponding "crater," while the former vortex transmutes into a stable fundamental $(2+1) \mathrm{D}$ soliton (with $m=0$ ), as seen in inset B (a detailed dynamical picture is provided in the movie in the Supplemental Material [18]).

The most remarkable manifestation of the spontaneous formation of complex patterns induced by the symmetry breaking above the effective threshold is the transmutation of vortices into various stars (see the right-hand-side column in Fig. 1), caused by small changes of $\Gamma$ and $\varepsilon$ in region S. How the circular symmetry is broken can be seen in detail in movies S1-S6 [18] that display the dynamics of the spontaneous emergence of four-, five-, six-, eight-, and ten-pointed stars, as well as of double-hump localized structures. For instance, at $\varepsilon=1.7$ and $\Gamma=0.018$, close to the junction of domains $\mathrm{C}$ and D, the input vortex [Fig. 2(a)] at first self-traps into a vortex soliton [Fig. 2(b)]. Then, the vortex loses its inner circular symmetry at $z \approx 140$, transforming into a crescent, while the phase dislocation continues to drift, until it escapes at $z \approx 730$ [18]. Therefore, the outer circular symmetry is gone too. At $z>900$, persistent oscillations commence, corresponding to a robust breather with period $T_{\mathrm{A}} \approx 5$ and a superimposed beat period, $T_{\mathrm{B}} \approx 40$. During another, much longer, beat period, $T_{\mathrm{M}} \approx 400$, the angular momentum oscillates between 

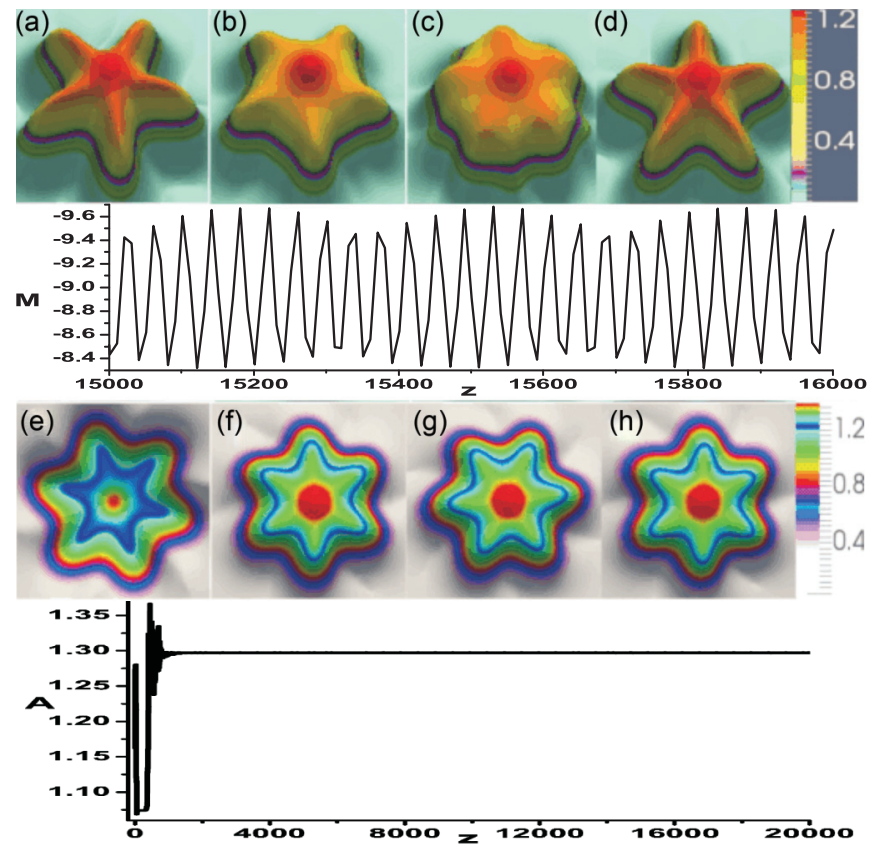

FIG. 3. (Color online) The evolution of the revolving five- and six-pointed stars. The five-pointed star at $z=1000$ (a) gets fatter from $z \approx 1010$ until $z \approx 1020$ (b) and (c), before rotating by $30^{\circ}$ at $z \approx 1040$ (d). The period of the cyclic evolution is $T_{\mathrm{C}} \approx 40$. Angular momentum $M$ oscillates between $M=-8.3$ and $M=-9.7$, with period $T_{\mathrm{M}} \approx 360$ (dimensionless units). (e) The transient six-pointed star self-traps into the permanent shape. (f)-(h) The rotation of the star in the course of $T / 6 \approx 36$ from $z \approx 1080$ until $z \approx 1116$ (only one sixth of the period is displayed, as the symmetry of the star makes the subsequent evolution tantamount to that shown here). Variations of amplitude $A$ in the course of the soliton self-formation are displayed in the bottom plot (dimensionless units).

$M=-0.18$ and $M=+0.18$ (see graph $\mathrm{M}$ in Fig. 2). In the course of beatings, a "Celtic-cross" shape transmutes into a sequence of crosslike ones [see Figs. 2(c)-2(g)], and eventually returns to a rotated "Celtic cross" [Fig. 2(h)] [18]. The robustness of the cyclic shape transmutations in this regime was tested up to $z=70000$. Reducing the gain curvature to $\Gamma=0.014$, at the same $\varepsilon=1.7$, increases the number of arms in the pattern, converting it into a five-pointed star, as shown in Figs. 3(a)-3(d) [18]. The scenario of breaking the axial symmetry to the reduced (fivefold) form is the same as in the previous case. However, the angular momentum now oscillates between $M=-8.3$ and $M=-9.7$, with period $T_{\mathrm{M}} \approx 360$ as in diagram $\mathrm{M}$ in Fig. 3. This star rotates and simultaneously changes its shape, with period $T_{\mathrm{C}} \approx 40$, between four particular five-pointed configurations.

Gradually decreasing the gain curvature $(\Gamma)$, and increasing the nonlinear gain $(\varepsilon)$, a sequence of six-, seven-, eight-, nine-, and ten-pointed stars is generated. After a transient period, each of them rotates at a constant angular velocity, keeping a permanent shape, unlike the cyclic "metamorphosis" featured by the four- and fivefold solitons. An example of a revolving six-pointed star (which resembles snowflakes [28]) is displayed in Figs. 3(e)-3(h), for $\Gamma=0.013$ and $\varepsilon=1.75$ (see movie in Supplemental Material [18]). The evolution of amplitude $A$ in the course of the soliton self-organization
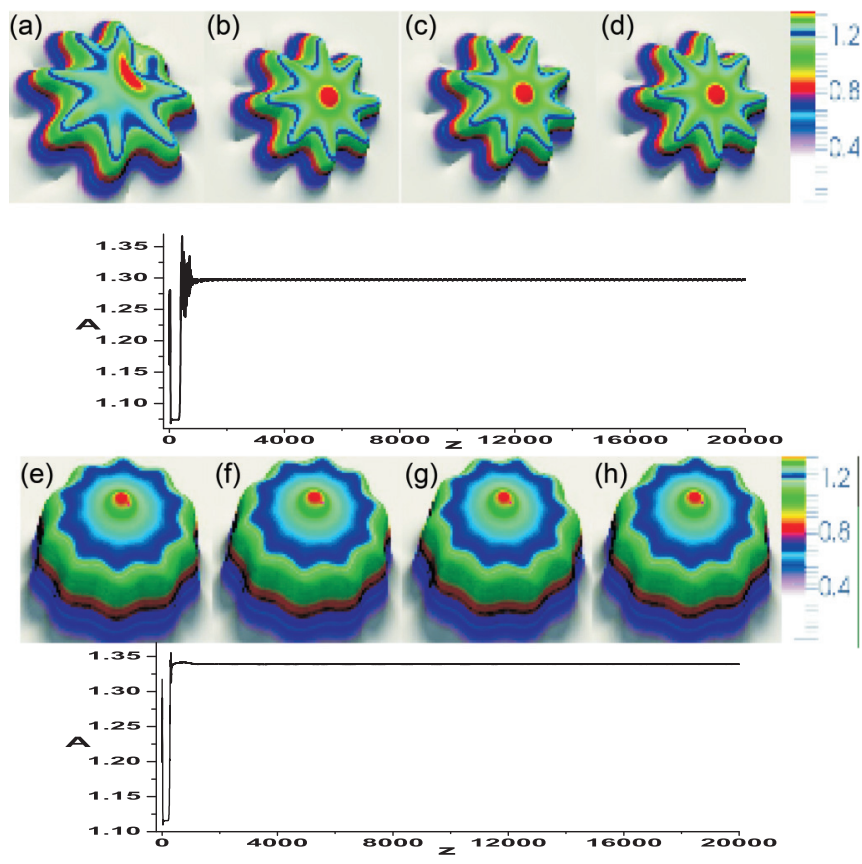

FIG. 4. (Color online) The evolution of the revolving eight- and ten-pointed stars. To establish the reduced symmetry, the phase dislocation is expelled from the vortical structure (a), and an "octopus" soliton emerges at $z \approx 960$ (b), which rotates, in the course of $T / 8 \approx 24$, through (c) at $z \approx 972$ into an identical configuration at $z \approx 984$ (d). Its spontaneous self-organization is illustrated by the bottom plot displaying the evolution of the amplitude, $A(z)$. (e)-(h) The rotation of a ten-pointed star ("decapod"), from (e) at $z \approx 1200$ to (h) at $z \approx 1248$ in the course of $T / 10 \approx 48$. Its self-generation is illustrated by the bottom plot for $A(z)$.

is presented in the same figure. In this case, the angular momentum, defined as per Eq. (3), is $M=-24$. Further, a typical eightfold "octopus" is presented in Figs. 4(a)-4(d), for the same $\varepsilon$ but a smaller gain curvature, $\Gamma=0.007$, featuring a smaller radius of the pattern's core [18]. The self-formation of the eight-pointed soliton star is shown in diagram A. Finally, the spontaneous self-organization of a ten-pointed star for $\varepsilon=1.95$ and $\Gamma=0.01$ is shown in Figs. 4(e)-4(h), and additionally illustrated by the plot for amplitude $A$ versus propagation distance $z$, which is displayed beneath panels 4(e)-4(h) [18]. All the stars are stable objects with a nonzero angular momentum but zero TC.

Structures with more than ten rays turn out to be transients evolving towards axially symmetric bell-shaped solitons in area B of Fig. 1. Lastly, at borderlines between B, S, and $\mathrm{U}$ areas there occur strongly asymmetric steadily rotating structures in the form of "cobras" (shown in the right bottom corner of Fig. 1).

It is precisely the spatially inhomogeneous linear gain $(\gamma>0)$ protruding above the loss sea, which drives, above an effective threshold, in synergy with the nonlinear gain, the spontaneous transition from vortices with topological charge $m=1$ to the various species of fundamental solitons with $m$ $=0$, including the above-mentioned class of the star-shaped solitons. Such a spontaneous transition is not possible with the gain submerged into the sea of loss, which was the distinctive 
feature of the model considered in Ref. [12]. Therefore, the two models are substantially different, although they include similar terms. As a result, in the model with $\gamma<0$, i.e., without the explicit linear gain, the stability chart in the same parameter plane is totally different [12].

It is relevant to stress the difference of the variety of patterns produced by the spontaneous breaking of the axial symmetry in systems of the CGLE type from their conservative counterparts, based on nonlinear Schrödinger equations (NLSEs). In the latter case, the angular momentum is always related to the TC, while the CGLE allows decoupling of the momentum from the TC, and $(2+1) \mathrm{D}$ NLSEs do not give rise to star-shaped patterns [29].

\section{CONCLUSIONS}

In conclusion, we have established that the evolution of the 2D complex Ginzburg-Landau equation with the cubic-quintic nonlinearity and localized linear gain gives rise to spontaneous formation of many species of patterns, including asymmetric vortices, modes which feature cyclic metamorphosis, and revolving stars without intrinsic TC (topological charge). Starting from the input provided by the variational approximation, systematic simulations have generated the localized structures whose stability areas are charted in Fig. 1 (only in a small part of the parameter space, the input decays without initiating the pattern formation). The instability-induced spontaneous reduction of the continuous rotational symmetry to a discrete subsymmetry is the generic route to the pattern formation in the present setting. In particular, the modulational instability breaks the vortex into two fragments, which fuse into a double-hump pattern above an effective threshold for the pattern formation, depending of the pump-beam intensity. More sophisticated species of the robust localized modes include four- and fivefold ones, which evolve through cycles of periodically changing forms. On the other hand, six-, seven-, eight-, nine-, and ten-pointed stars steadily revolve, keeping the constant shape and constant angular momentum, with zero $\mathrm{TC}$, unlike vortices, whose angular momentum is proportional to the TC. Stable, oddly shaped rotating patterns (cobras) occur in interstices between stability domains of the different species.

The proposed $(2+1) \mathrm{D}$ CGLE model establishes the complex pattern-formation phenomenology in laser cavities and other nonlinear photonic systems. As a consequence, it may be used to monitor real-time stability, and to detect fluctuations causing slight changes of the cavity $(\varepsilon)$ and pump $(\Gamma)$ control parameters in operating lasers, that can be visualized by the change of patterns (e.g., the increase of the number of arms in the star structures). The model may also help in understanding generic features of the pattern formation in other areas, with potential applications to the design of information-processing analog schemes [30].

\section{ACKNOWLEDGMENTS}

This work was supported, in part, by the Ministry of Science of Serbia through Projects No. OI 171006 and No. III 45016. This publication was partially made possible by NPRP Grants No. 5-674-1-114 and No. 6-021-1-005 from the Qatar National Research Fund (a member of Qatar Foundation). D.M. was supported by the Romanian Ministry of Education and Research (Project No. PN-II-ID-PCE-2011-3-0083).
[1] G. Nicolis and I. Prigogine, Self-Organization in NonEquilibrium Systems (Wiley, New York, 1977).

[2] I. S. Aranson and L. Kramer, Rev. Mod. Phys. 74, 99 (2002); N. Rosanov, Spatial Hysteresis and Optical Patterns (Springer, Berlin, 2002); Dissipative Solitons: From Optics to Biology and Medicine, Lecture Notes in Physics Vol. 751, edited by N. N. Akhmediev and A. Ankiewicz (Springer, Berlin, 2008).

[3] B. A. Malomed, Physica D 29, 155 (1987); S. Fauve and O. Thual, Phys. Rev. Lett. 64, 282 (1990); W. van Saarloos and P. C. Hohenberg, ibid. 64, 749 (1990); V. Hakim, P. Jakobsen, and Y. Pomeau, Europhys. Lett. 11, 19 (1990); B. A. Malomed and A. A. Nepomnyashchy, Phys. Rev. A 42, 6009 (1990); A. Komarov, H. Leblond, and F. Sanchez, Phys. Rev. E 72, 025604 (2005); J. N. Kutz, SIAM Rev. 48, 629 (2006); W. H. Renninger, A. Chong, and F. W. Wise, Phys. Rev. A 77, 023814 (2008); E. Ding and J. N. Kutz, J. Opt. Soc. Am. B 26, 2290 (2009); M. Tlidi, A. G. Vladimirov, D. Pieroux, and D. Turaev, Phys. Rev. Lett. 103, 103904 (2009); D. Mihalache, Proc. Rom. Acad. Ser. A 11, 142 (2010); J. Jimenez, Y. Noblet, P. V. Paulau, D. Gomila, and T. Ackemann, J. Opt. 15, 044011 (2013); C. Fernandez-Oto, M. G. Clerc, D. Escaff, and M. Tlidi, Phys. Rev. Lett. 110, 174101 (2013).

[4] P. W. Anderson, Science 177, 393 (1972).
[5] A. M. Turing, Philos. Trans. R. Soc. London 237, 37 (1952).

[6] P. Tracqui, Rep. Prog. Phys. 72, 056701 (2009); S. Kondo and T. Miura, Science 329, 1616 (2010).

[7] M. Eiraku, N. Takata, H. Ishibashi, M. Kawada, E. Sakakura, S. Okuda, K. Sekiguchi, T. Adachi, and Y. Sasai, Nature 472, 51 (2011).

[8] Y. S. Kivshar and G. P. Agrawal, Optical Solitons: From Fibers to Photonic Crystals (Academic Press, San Diego, 2003).

[9] F. T. Arecchi, S. Boccaletti, and P. L. Ramazza, Phys. Rep. 318, 83 (1999); F. Lederer, G. I. Stegeman, D. N. Christodoulides, G. Assanto, M. Segev, and Y. Silberberg, ibid. 463, 1 (2008).

[10] D. Mihalache, D. Mazilu, F. Lederer, Y. V. Kartashov, L.-C. Crasovan, L. Torner, and B. A. Malomed, Phys. Rev. Lett. 97, 073904 (2006).

[11] V. Skarka and N. B. Aleksić, Phys. Rev. Lett. 96, 013903 (2006).

[12] V. Skarka, N. B. Aleksić, H. Leblond, B. A. Malomed, and D. Mihalache, Phys. Rev. Lett. 105, 213901 (2010).

[13] E. L. Falcao-Filho, C. B. de Araujo, G. Boudebs, H. Leblond, and V. Skarka, Phys. Rev. Lett. 110, 013901 (2013).

[14] V. Skarka, N. B. Aleksić, and V. I. Berezhiani, Phys. Rev. A 81, 045803 (2010).

[15] N. N. Akhmediev and A. Ankiewicz, Solitons, Nonlinear Pulses and Beams (Chapman and Hall, London, 1997). 
[16] B. A. Malomed and H. G. Winful, Phys. Rev. E 53, 5365 (1996); P. V. Paulau, ibid. 84, 036213 (2011).

[17] L.-C. Crasovan, B. A. Malomed, and D. Mihalache, Phys. Rev. E 63, 016605 (2001); V. Skarka, N. B. Aleksić, M. Derbazi, and V. I. Berezhiani, Phys. Rev. B 81, 035202 (2010).

[18] See Supplemental Material at http://link.aps.org/supplemental/ 10.1103/PhysRevA.90.023845 for Movies S1-S6 which display the real dynamics of the self-organized pattern formation of four-, five-, six-, eight-, and ten-pointed stars, as well as of the double-hump structures (with the same color bars as in corresponding figures).

[19] A. S. Desyatnikov and Yu. S. Kivshar, Phys. Rev. Lett. 88, 053901 (2002); A. S. Desyatnikov, A. A. Sukhorukov, and Y. S. Kivshar, ibid. 95, 203904 (2005); V. M. Lashkin, E. A. Ostrovskaya, A. S. Desyatnikov, and Y. S. Kivshar, Phys. Rev. A 80, 013615 (2009).

[20] H. Sakaguchi and B. A. Malomed, J. Phys. B: At. Mol. Opt. Phys. 37, 2225 (2004).

[21] T. Y. Fan, A. Sanchez, and W. E. DeFeo, Opt. Lett. 14, 1057 (1989); W. Koechner and M. Bass, Solid-State Lasers: A Graduate Text (Springer, New York, 2003).

[22] F. Salin, J. Squier, and M. Piche, Opt. Lett. 16, 1674 (1991); X. Yan, Q. Liu, D. Wang, and M. Gong, Opt. Express 19, 6883 (2011); Z. Xiang, D. Wang, S. Pan, Y. Dong, Z. Zhao, T. Li, J.
Ge, Ch. Liu, and J. Chen, ibid. 19, 21060 (2011); P. R. Battle, J. G. Wessel, and J. L. Carlsten, Phys. Rev. A 48, 707 (1993); J. E. La Sala, D. A. G. Deacon, and J. M. J. Madey, Phys. Rev. Lett. 59, 2047 (1987); E. E. Fill, Opt. Commun. 67, 441 (1988).

[23] F. Salin and J. Squier, Opt. Lett. 17, 1352 (1992).

[24] N. N. Akhmediev, V. V. Afanasjev, and J. M. Soto-Crespo, Phys. Rev. E 53, 1190 (1996); J. Atai and B. A. Malomed, Phys. Lett. A 246, 412 (1998); W. J. Firth and P. V. Paulau, Eur. Phys. J. D 59, 13 (2010).

[25] D. J. Kaup and B. A. Malomed, Physica D 87, 101 (1995).

[26] V. Skarka, D. V. Timotijević, and N. B. Aleksić, J. Opt. A: Pure Appl. Opt. 10, 075102 (2008).

[27] B. N. Aleksić, N. B. Aleksić, V. Skarka, and M. Belić, Phys. Scr. T 149, 014036 (2012).

[28] T. Bartels-Rausch, V. Bergeron, J. H. E. Cartwright, R. Escribano, J. L. Finney, H. Grothe, P. P. J. Gutiérrez, J. Haapala, W. F. Kuhs, J. B. C. Pettersson, S. D. Price, C. I. Sainz-Díaz, D. J. Stokes, G. Strazzulla, E. S. Thomson, H. Trinks, and N. Uras-Aytemiz, Rev. Mod. Phys. 84, 885 (2012).

[29] B. A. Malomed, D. Mihalache, F. Wise, and L. Torner, J. Opt. B: Quantum Semiclassical Opt. 7, R53 (2005).

[30] Spontaneous Symmetry Breaking, Self-Trapping, and Josephson Oscillations, edited by B. A. Malomed (Springer-Verlag, Berlin and Heildelberg, 2013). 